\title{
El efecto del pastoreo sobre la diversidad florística y estructural en pastizales de montaña del centro de Argentina
}

\author{
Grazing effects on the floristic and structural diversity in mountain grasslands \\ from central Argentina
}

\begin{abstract}
MARIANA NAI-BREGAGLIO ${ }^{1}$, EDUARDO PUCHETA $^{1,2} \&$ MARCELO CABIDO $^{1}$
${ }^{1}$ Facultad de Ciencias Exactas, Físicas y Naturales e Instituto Multidisciplinario de Biología Vegetal, Universidad Nacional de Córdoba, Casilla de Correos 495, 5000 Córdoba, Argentina

${ }^{2}$ Departamento de Geofísica, Astronomía y Biología, Facultad de Ciencias Exactas, Físicas y Naturales, Universidad Nacional de San Juan, Argentina; e-mail: epucheta@com.uncor.edu
\end{abstract}

\begin{abstract}
RESUMEN
Se estudió el efecto del pastoreo y de su exclusión por 10 años sobre la composición y diversidad florística y sobre la diversidad estructural de pastizales naturales de montaña, en las Sierras de Córdoba (2.200 m de altitud), Argentina. Consideramos diversidad estructural a la complejidad en la disposición de la biomasa en el espacio horizontal y vertical. Se estimó la abundancia de todas las especies de plantas y su arreglo en el espacio vertical y horizontal, por medio de 50 agujas distribuidas al azar, subdivididas en intervalos verticales de $20 \mathrm{~cm}$. El muestreo se realizó en el interior de cinco réplicas, cada una de las cuales consistió de dos parcelas adyacentes de 20 × $20 \mathrm{~m}$ separadas por un alambrado, una pastoreada y otra excluida al pastoreo. Se estimaron índices de diversidad de Shannon para la composición florística y para la estructura vertical. Para analizar las posibles variaciones entre tratamientos en la distribución horizontal de la diversidad de especies y de la diversidad de estratos verticales, se compararon las varianzas de los índices de diversidad florística y de la estructura vertical, respectivamente. La riqueza de especies y la diversidad florística alcanzaron valores máximos en sitios pastoreados. Más del $85 \%$ del total de especies se registraron en estos sitios en contraste con un $65 \%$ presente en los sitios excluidos al ganado. Sólo se registraron tres especies de plantas exóticas, una de ellas exclusiva de los sitios pastoreados. El pastoreo produjo una disminución significativa de la diversidad de estratos verticales y de la varianza en el espacio horizontal del índice de diversidad florística, pero no produjo cambios significativos en la varianza en el espacio horizontal del índice de diversidad de estratos verticales. Si bien el pastoreo promueve la formación de céspedes de alta diversidad florística, produce, además, una simplificación de la estructura comunitaria, tanto en sentido vertical como horizontal, afectando probablemente a otros niveles tróficos del ecosistema. La alternancia en el espacio de los dos tipos de parches estudiados permitiría obtener un mejor compromiso entre pastoreo y conservación de la diversidad florística y estructural.
\end{abstract}

Palabras clave: estructura horizontal, estructura vertical, exclusión, pastoreo, diversidad.

\begin{abstract}
The effects of grazing and 10 years of cattle exclusion on floristic composition and diversity and on structural diversity were studied in natural mountain grasslands at Sierras de Córdoba (2,200 m of elevation), Argentine. We consider structural diversity as the arrangement complexity of biomass in space, both horizontal and vertical. The abundance of all plant species and their vertical and horizontal arrangement were recorded by means of 50 randomly assorted pins, each of them subdivided into $20 \mathrm{~cm}$ height intervals. Sampling was performed inside five replicates, each of them consisted of two adjacent $20 \times 20 \mathrm{~m}$ plots separated by a fence, one grazed and another excluded from grazing. Floristic and vertical structure diversity were estimated by means of Shannon index. The variance in floristic and vertical structure diversity indexes were compared among treatments to study possible variations in the horizontal distribution of both floristic diversity and vertical structure, respectively. Species richness and floristic diversity were higher in grazed grasslands. Over $85 \%$ of total plant species occurred at grazed grasslands, in contrast to $65 \%$ in sites excluded from grazing. Only three exotic plant species were found, one of them exclusive to grazed sites. Cattle grazing produced a significant decrease in vertical structure diversity and in the variance in horizontal space of floristic diversity, but did not produce significant changes in the variance in horizontal space of vertical structure diversity. Although cattle grazing promotes lawns of high floristic diversity, it produces an over simplification of the community structure, both vertical and horizontal, probably affecting other trophic levels of the ecosystem. We consider that the mixture in space of both types of patches studied in the present work would allow a better balance between cattle grazing and conserving floristic and structural diversity.
\end{abstract}

Key words: diversity, grazing, grazing exclusion, horizontal structure, vertical structure. 


\section{INTRODUCCIÓN}

La influencia de los grandes herbívoros sobre la estructura de la vegetación en pastizales es compleja, ya que no sólo remueven una gran proporción de la biomasa aérea, sino que producen efectos directos e indirectos sobre la dispersión, el establecimiento, el crecimiento y la reproducción de las plantas (Belsky 1986, Collins 1987).

Entre los efectos más comunes que produce el pastoreo sobre la estructura de pastizales naturales, están los cambios en la diversidad florística (Milchunas \& Lauenroth 1993, Pettit et al. 1995, Pucheta et al. 1998) y en la diversidad estructural de la comunidad (Sala et al. 1986, Milchunas \& Lauenroth 1989, Molina et al. 1999). Si bien la diversidad estructural de una comunidad hace referencia no sólo a la organización de su biomasa en el espacio en un sitio, sino también a la presencia y abundancia de determinadas especies (Magurran 1988), en este trabajo definimos diversidad estructural a la disposición de la biomasa en el espacio horizontal y vertical. Estos componentes de la diversidad no han sido tenidos en cuenta en la mayoría de estudios relacionados con el pastoreo u otras perturbaciones, a pesar de su enorme importancia para otros niveles tróficos del sistema (Sala et al. 1986, Gardner et al. 1995, Molina et al. 1999).

El pastoreo por ungulados nativos es considerado como el principal responsable de mantener una alta diversidad de especies en pastizales naturales, debido al consumo que ejercen sobre las especies dominantes de la comunidad (Milchunas et al. 1988, Perevolotsky \& Seligman 1998, Knapp et al. 1999). Además, la presencia o incorporación de herbívoros ha comenzado a significar un importante componente en planes de manejo para la mantención y restauración de la diversidad, particularmente en pastizales naturales (Olff \& Ritchie 1998). Sin embargo, la diversidad de especies no siempre es incrementada por el pastoreo y depende, entre otras cosas, del tipo de herbívoros y de la intensidad de la herbivoría (Milchunas \& Lauenroth 1993), de la escala espacial (Hobbs \& Huenneke 1992) o temporal considerada (Anderson \& Briske 1995), o de las condiciones del suelo y el clima (Milchunas et al. 1988, Hobbs \& Huenneke 1992).

El pastoreo es considerado una perturbación en algunos pastizales de Argentina (León et al. 1984, Sala et al. 1986, Facelli et al. 1988) y de Australia (Wilson 1990, McIntyre \& Lavorel 1994). Sin embargo, en otros pastizales el pastoreo es considerado un componente incorporado al ecosistema (Milchunas et al. 1988, Noy-Meir et al. 1989, Díaz et al. 1994a, Perevolotsky \& Seligman 1998,
Pucheta et al. 1998, Knapp et al. 1999). En efecto, las respuestas estructurales y funcionales de los pastizales naturales frente a la herbivoría pueden ser muy diferentes y a veces opuestas, debido a diferencias en su historia de pastoreo (Milchunas et al. 1988, Milchunas \& Lauenroth 1993). En pastizales con corta historia de pastoreo, como los pastizales pampeanos de Argentina, o los pastizales templados de Australia, el pastoreo produce una disminución de la diversidad a cargas moderadas y extinciones locales de algunas especies nativas; además, a cargas moderadas a intensas puede favorecer la invasión de especies exóticas. En pastizales con larga historia de pastoreo, en cambio, la diversidad es máxima en presencia de los herbívoros y en general, no se observa la invasión de especies exóticas ni la pérdida de especies nativas aún a una alta carga ganadera (Milchunas et al. 1988, Noy-Meir et al. 1989).

Los pastizales de montaña de las Sierras de Córdoba han estado sujetos al pastoreo de camélidos (Lama guanicoe, Vicugna vicugna) durante gran parte del Pleistoceno y de animales domésticos, como vacas, caballos y mulas, desde la ocupación española hace más de 300 años (Díaz et al. 1994a), y soportan actualmente un régimen de ganadería extensiva, con cargas moderadas a altas de ganado vacuno. Algunas respuestas de la comunidad vegetal frente a la acción de los herbívoros domésticos han sido estudiadas previamente en el área, como cambios en su fenología (Díaz et al. 1994b), en su composición florística (Pucheta \& Cabido 1992, Pucheta et al. 1992, 1998), en su biomasa y productividad primaria neta aérea (Pucheta et al. 1998).

El presente trabajo pretende evaluar cuál es el efecto del pastoreo por ganado doméstico y su exclusión prolongada, sobre la estructura de la comunidad vegetal al nivel de (1) su diversidad florística y (2) su diversidad estructural, ésta última estimada como la distribución de la biomasa en el espacio vertical y horizontal. Interesa conocer de qué manera se puede alcanzar un compromiso entre la cría de ganado doméstico, la conservación de la diversidad de especies nativas y la conservación de la diversidad estructural del pastizal, ambos aspectos de la diversidad que contribuyen a una mayor disponibilidad de hábitats para otros niveles tróficos.

\section{MATERIALES Y MÉTODOS}

\section{Área de estudio}

Está ubicada en la altiplanicie granítica de Pampa de Achala, Sierras de Córdoba, a 2.200 m; abarca 
una faja orientada en sentido N-S de unos $60 \mathrm{~km}$ de longitud $\left(31^{\circ} 24^{\prime}-31^{\circ} 50^{\prime} \mathrm{S}\right)$ y $8 \mathrm{~km}$ en su parte más ancha $\left(64^{\circ} 45^{\prime}-64^{\circ} 52^{\prime} \mathrm{O}\right)$. El clima es típicamente de montaña, con temperaturas bajas en invierno, grandes variaciones térmicas, fuertes vientos, lluvias concentradas en la estación más cálida, heladas frecuentes durante gran parte del año y algunas nevadas (Cabido 1985). Las temperaturas medias de invierno y verano son de 5 y $11,4{ }^{\circ} \mathrm{C}$, respectivamente. Las precipitaciones alcanzan un valor promedio de $850 \mathrm{~mm}$ para 12 años de registros (Cabido 1985).

La Pampa de Achala se encuentra ubicada fitogeográficamente en el subpiso superior de pastizales de altura de las Sierras de Córdoba (Luti et al. 1979) dentro del Distrito Chaqueño Serrano (Cabrera 1976).

El presente estudio se concentró en dos de los diversos estados sucesionales que pueden presentar los pastizales naturales de las Sierras Grandes (Pucheta et al. 1997), comprendidos por los pajonales de Deyeuxia hieronymi y Festuca tucumanica, y por los céspedes de pastoreo de Alchemilla pinnata, Carex fuscula y Relbunium richardianum.

\section{Diseño del muestreo}

Para evaluar el efecto del pastoreo sobre la diversidad estructural de los pastizales naturales del área de estudio se tuvieron en cuenta dos tratamientos, uno pastoreado y otro excluido al pastoreo por 10 años. Los tratamientos se evaluaron por medio de cinco réplicas separadas unas de otras unos $4 \mathrm{~km}$, cada una de las cuales consistió en dos parcelas adyacentes de $20 \times 20 \mathrm{~m}$ separadas por un alambrado, una parcela pastoreada y otra excluida al pastoreo. El tratamiento pastoreado posee una carga moderada a intensa (1 res 3-4 ha-1) por lo menos durante los últimos 10 años, mientras que el tratamiento excluido al pastoreo, representa sitios donde el ganado fue retirado luego del último trazado de la ruta nacional No. 20 en 1987 y constituyen exclusiones naturales a lo largo del camino, situación similar a la utilizada por NoyMeir et al. (1989). Las parcelas a uno y otro lado del alambrado (cinco pares) comparten las mismas condiciones abióticas desde el ensanche del camino e instalación del alambrado y se encuentran libres de incendio desde entonces.

Para estimar el efecto del pastoreo sobre la composición florística y la diversidad de especies, se registró la presencia y la abundancia (número de contactos) de todas las especies de plantas en 50 agujas distribuidas al azar en cada una de las réplicas. Para evaluar el efecto del pastoreo sobre la estructura vertical del pastizal se registró la abundancia de la vegetación independientemente de la taxonomía de las especies (número de contactos por aguja), teniendo en cuenta ocho diferentes intervalos de altura (0-5, 5-10, 10-20, 20-30, 30-40, 40-60, 60-80, y 80$100 \mathrm{~cm}$ ). Para evaluar el efecto del pastoreo sobre la abundancia de estratos y la abundancia de las especies en sentido horizontal, se registró la abundancia de plantas por aguja y por estrato independientemente de la identidad taxonómica de las especies y la abundancia de cada una de las especies por aguja sin tener en cuenta los intervalos de altura. El muestreo se realizó entre los meses de marzo y abril de 1997.

\section{Análisis de datos}

Para determinar las principales tendencias de variación florística de las réplicas en pastizales pastoreados y excluidos al pastoreo, se realizó un análisis de ordenación (DCA, Hill \& Gauch 1981) con los valores de abundancia de las especies presentes. Los datos florísticos se presentan en una tabla ordenada que incluye las especies de los 10 sitios relevados, cinco pastoreados y cinco excluidos al pastoreo.

Para estimar la diversidad florística y diversidad de estratos verticales se utilizó el índice de Shannon (Magurran 1988). Para estimar la variación de la diversidad de estratos verticales y de la diversidad florística en el espacio horizontal, se realizaron comparaciones de la varianza entre los índices de ambos tratamientos. Las diferencias entre tratamientos en los índices de diversidad (florística y vertical) y la comparación de la varianzas de esos índices se evaluaron a través de pruebas no paramétricas de Kruskal-Wallis y Kolmogorov-Smirnov mediante el programa SPSS (Norušis 1992).

Para determinar las posibles diferencias entre tratamientos en las relaciones de dominancia de las especies que componen el pastizal, se elaboraron curvas de dominancia-diversidad a través de los valores medios de abundancia de las especies registradas en todas las réplicas.

\section{RESULTADOS}

Efectos del pastoreo sobre la composición florística y la diversidad

El análisis de ordenamiento (DCA) de las réplicas de los tratamientos en función de su composición 
de especies, muestra importantes diferencias florísticas entre las parcelas de los tratamientos pastoreado y excluido por 10 años (Fig. 1). Se registraron un total de 70 especies de Angiospermas y dos especies de Pteridófitos en los pastizales estudiados, 64 de ellas en los sitios pastoreados y 46 en los sitios excluidos al pastoreo (Anexo 1).

En el tratamiento pastoreado se encontraron 25 especies de plantas que no estaban en la comunidad vegetal en ausencia prolongada del pastoreo y se registró un incremento en la abundancia de otras 10 especies de plantas (Anexo 1). Una importante cantidad de especies (29) se encontró en ambos tratamientos, algunas de ellas no disminuyeron drásticamente su abundancia con el pastoreo, a pesar de ser especies seleccionadas por el ganado doméstico (Sorghastrum pellitum, Carex spp., Bromus auleticus, Briza subaristata) (V. Falczuk, comunicación personal). Otras siete especies sólo se encontraron en ausencia prolongada del ganado, pero no parecen estar relacionadas con el consumo de los herbívoros, ya que no son especies muy palatables (V. Falczuk, comunicación personal) (Anexo 1).

Se registraron tres especies de plantas exóticas de amplia distribución, una de ellas asociada sólo a pastizales pastoreados (Poa annua), mientras que las restantes se encontraron en ambos tratamientos (Rumex acetosella y Taraxacum officinale). La diversidad de especies fue significativamente mayor $(\mathrm{P}=0,009)$ en los sitios pastoreados $\left(\mathrm{H}^{\prime}=3,60 \pm 0,19\right)$ en relación con los sitios excluidos al ganado $\left(\mathrm{H}^{\prime}=1,28 \pm 0,11\right)$. Los valores de abundancia de las diferentes especies en ambos tratamientos permiten visualizar las relaciones de dominancia entre ellas (Fig. 2). Los valores de abundancia de las especies dominantes del tratamiento excluido al ganado por 10 años presentan un orden de magnitud superior en relación a otras especies del mismo tratamiento y en relación a las especies más comunes del tratamiento pastoreado (Fig. 2). La pendiente de la curva en el tratamiento pastoreado es menos pronunciada, evidenciando en estos sitios una mayor equitatividad de las especies.

En los sitios pastoreados, las especies más abundantes son Muhlenbergia peruviana, Alchemilla pinnata, Carexfuscula y Relbunium richardianum, todas especies graminoides o herbáceas rastreras de pequeña estatura, mientras que en los sitios excluidos al ganado por más de 10 años, las especies más abundantes son Deyeuxia hieronymi, Poa stuckertii y Festuca tucumanica, especies de gramíneas de gran estatura.

Efectos del pastoreo sobre la diversidad vertical y horizontal de la comunidad

El pastoreo produjo un importante efecto simplificador de la estructura vertical del pastizal

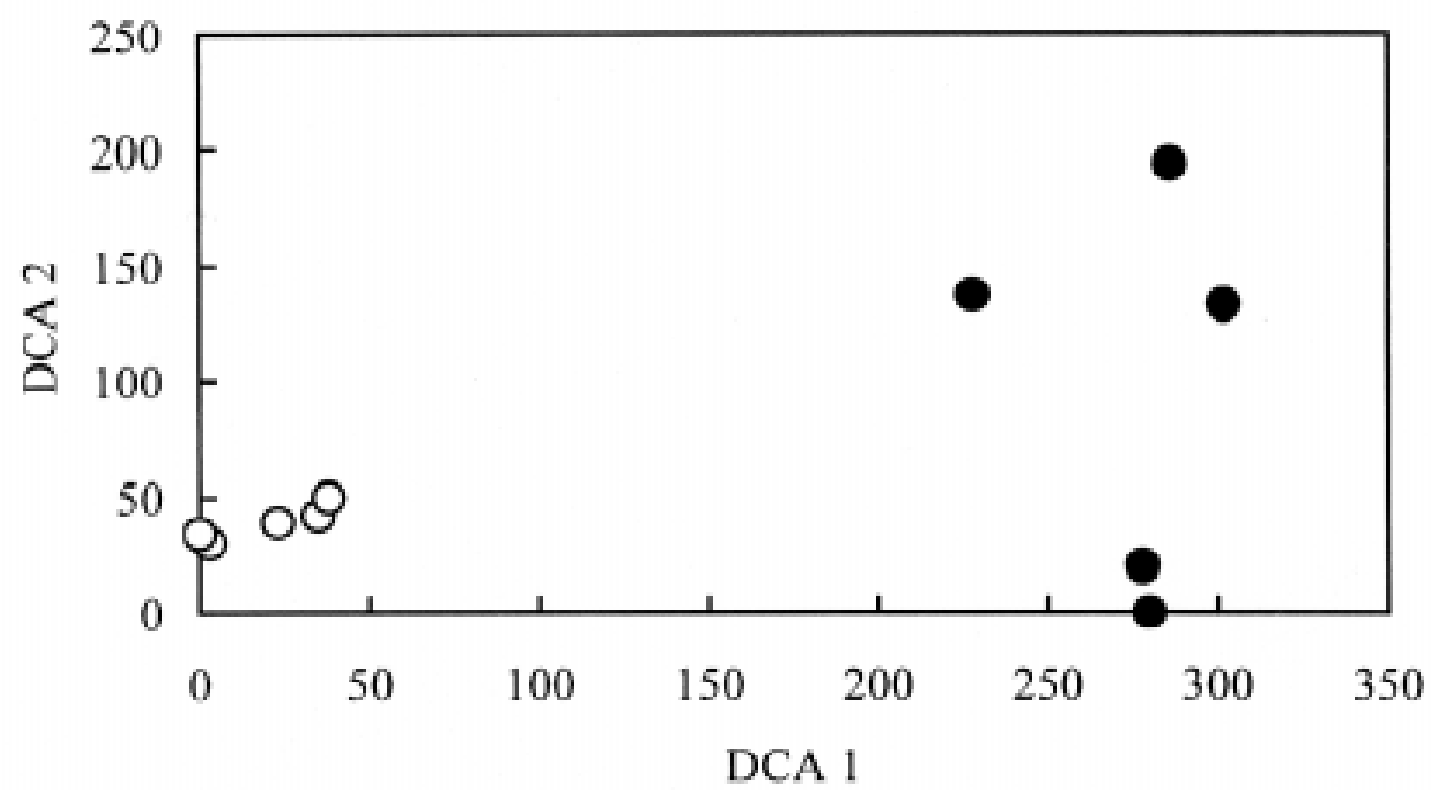

Fig. 1: Ordenamiento (DCA) de las réplicas de ambos tratamientos en función de la presencia y abundancia de las especies, en pastizales de montaña de las Sierras de Córdoba, Argentina. Círculos blancos: tratamiento pastoreado; círculos negros: tratamiento excluido al pastoreo los últimos 10 años.

Replicate ordination (DCA) in relation to species presence and abundance, in mountain grasslands of Sierras de Córdoba, Argentina. Open circles: grazed treatment; filled circles: exclusion from grazing the last 10 years. 


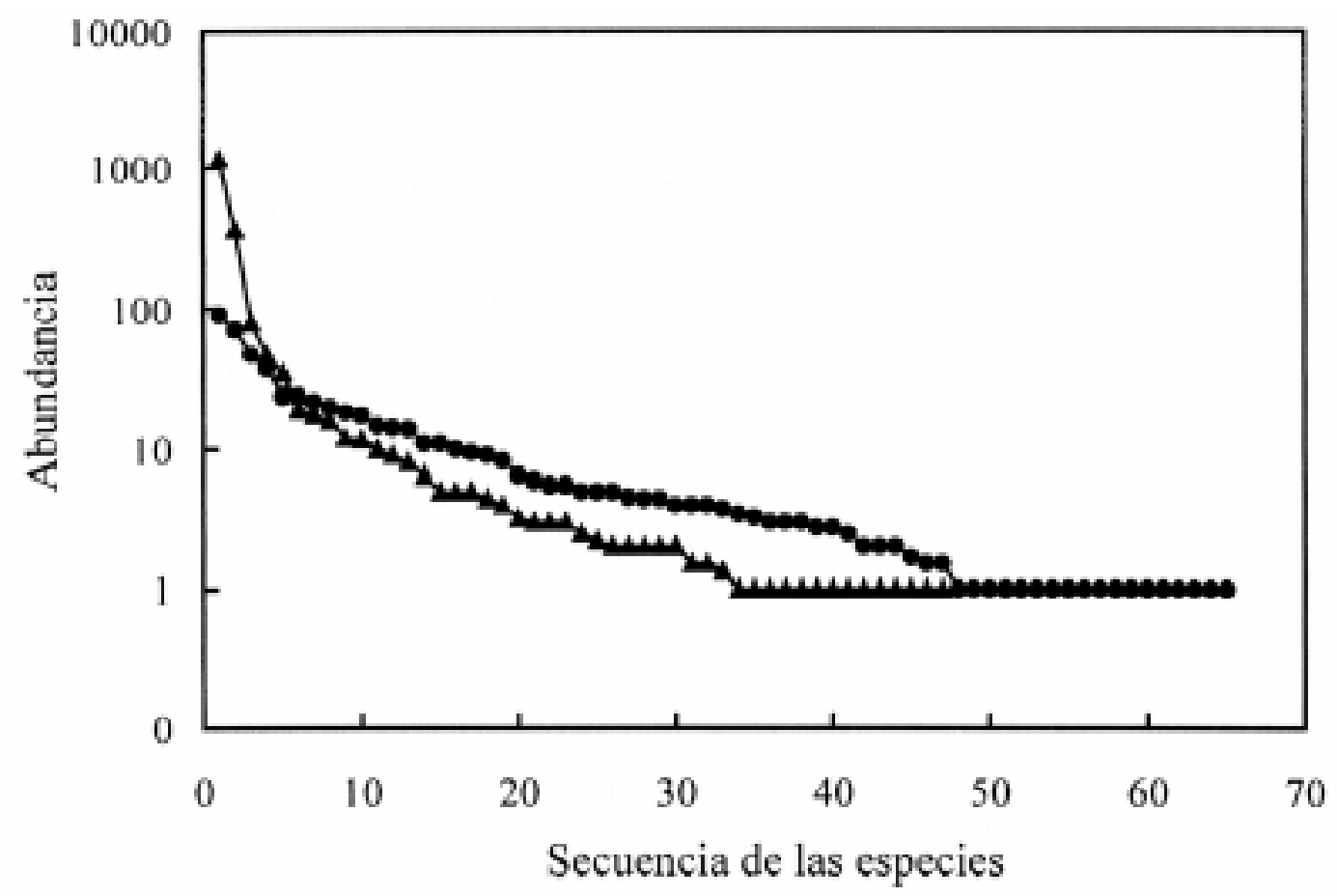

Fig. 2: Curvas de dominancia-diversidad para los tratamientos pastoreado (círculos) y excluido al pastoreo los últimos 10 años (triángulos), en pastizales de montaña de las Sierras de Córdoba, Argentina. Nótese la escala logarítmica en eje de ordenadas.

Dominance-diversity curves for grazed (circles) and 10 years cattle exclusion (triangles) treatments, in mountain grasslands of Sierras de Córdoba, Argentina. Note the logarithmic scale in ordinates axis.

(Fig. 3). En los sitios pastoreados el 77,4 \% de la biomasa de las especies se concentró en el primer intervalo de altura, entre el nivel del suelo y los primeros $5 \mathrm{~cm}$, siendo de $40 \mathrm{~cm}$ la altura máxima registrada (Fig. 3). La exclusión prolongada del ganado, por el contrario, permitió un mayor desarrollo de la biomasa en el espacio vertical. En este tratamiento, el $70 \%$ de la biomasa de las especies se encontró por encima de los $20 \mathrm{~cm}$ de altura (Fig. 3).

En el tratamiento excluido al ganado, el índice de diversidad de estratos verticales fue significativamente mayor $\left(\mathrm{H}^{\prime}=2,56 \pm 0,03 ; \mathrm{P}=\right.$ $0,009)$ en relación al tratamiento pastoreado $\left(\mathrm{H}^{\prime}\right.$ $=0,94 \pm 0,16)$. El pastoreo, por otra parte, no produjo una modificación significativa en los patrones de distribución horizontal de estos estratos verticales $(P=0,251)$, en relación con los sitios excluidos al pastoreo, y produjo una distribución más homogénea de la diversidad de especies en sentido horizontal $(\mathrm{P}=0,0001)$.

\section{DISCUSIÓN}

En los pastizales estudiados, el pastoreo por ganado doméstico produjo un incremento de la ri- queza y de la diversidad de especies, pero provocó una simplificación de la estructura vertical y horizontal. En sitios pastoreados se registró una mayor riqueza de especies nativas. Si bien, ambos tratamientos compartieron el $54 \%$ de las especies, en los sitios pastoreados se encontró más del $85 \%$ del total de las especies registradas, en contraste con un $65 \%$ de las especies registradas en los sitios excluidos al pastoreo los últimos 10 años. Una mayor riqueza de especies nativas en presencia de grandes herbívoros se ha observado en pastizales muy similares a los del estudio (Pucheta \& Cabido 1992, Pucheta et al. 1992, 1998), en otros pastizales subhúmedos de diversos sitios del mundo (Milchunas et al. 1988, Rambo \& Faeth 1999, Karki et al. 2000) y en sistemas de pastizales mediterráneos (Noy-Meir et al. 1989, Montalvo et al. 1993).

Muy pocas especies (menos del $10 \%$ ) desaparecieron bajo la presión del ganado doméstico, ninguna de las cuales es elegida por los herbívoros (V. Falczuk, comunicación personal). Por el contrario, las especies más elegidas por el ganado (V. Falczuk, comunicación personal) no se vieron afectadas (Carex spp., Briza subaristata, Trifolium amabile, Sorghastrum pellitum), o aún aumentaron sus valores de abundancia bajo el pastoreo 
(Eleocharis albibracteata, Sisyrrinchium spp., Agrostis spp.). Sólo se registraron tres especies de plantas exóticas cosmopolitas, una de ellas asociada a pastizales pastoreados y otras dos ubicuas. El aumento de la riqueza de especies nativas y la baja invasibilidad por especies exóticas, han sido observados en diversos pastizales pastoreados con una larga historia de herbivoría o con presiones de selección similares o convergentes con el pastoreo (Milchunas et al. 1988, Milchunas \& Lauenroth 1993). Por otro lado, la invasión del pastizal por especies exóticas frente al pastoreo representa una respuesta comunitaria muy común en pastizales de la pampa húmeda de Argentina (Sala et al. 1986, Chaneton \& Facelli 1991) y de Australia (McIntyre \& Lavorel 1994, Pettit et al. 1995). La baja invasibilidad de los pastizales estudiados representa un caso particular dentro de los pastizales del centro de Argentina, en relación a su respuesta frente al pastoreo.
El incremento de la diversidad de especies frente al pastoreo observado en el presente estudio puede explicarse a través de una disminución de la dominancia de algunas pocas especies de gramíneas de gran estatura (Deyeuxia hieronymi, Poa stuckertii y Festuca tucumanica) como se ha observado en pastizales similares a los estudiados (Pucheta \& Cabido 1992, Pucheta et al. 1992, 1998) y en otros pastizales naturales o semi naturales (Milchunas et al. 1988, Chaneton \& Facelli 1991, Perevolotsky \& Seligman 1998, Knapp et al. 1999).

El pastoreo produjo una simplificación de la diversidad de estratos verticales, concentrando la biomasa dentro de los primeros $5 \mathrm{~cm}$ de altura promoviendo, además, una mayor uniformidad en la distribución de las especies en el espacio horizontal. Estos cambios implicarían una disminución de la diversidad de hábitats para otros niveles tróficos, como pequeños herbívoros e insectos sobre (Gardner et al. 1995, Molina et al. 1999) o

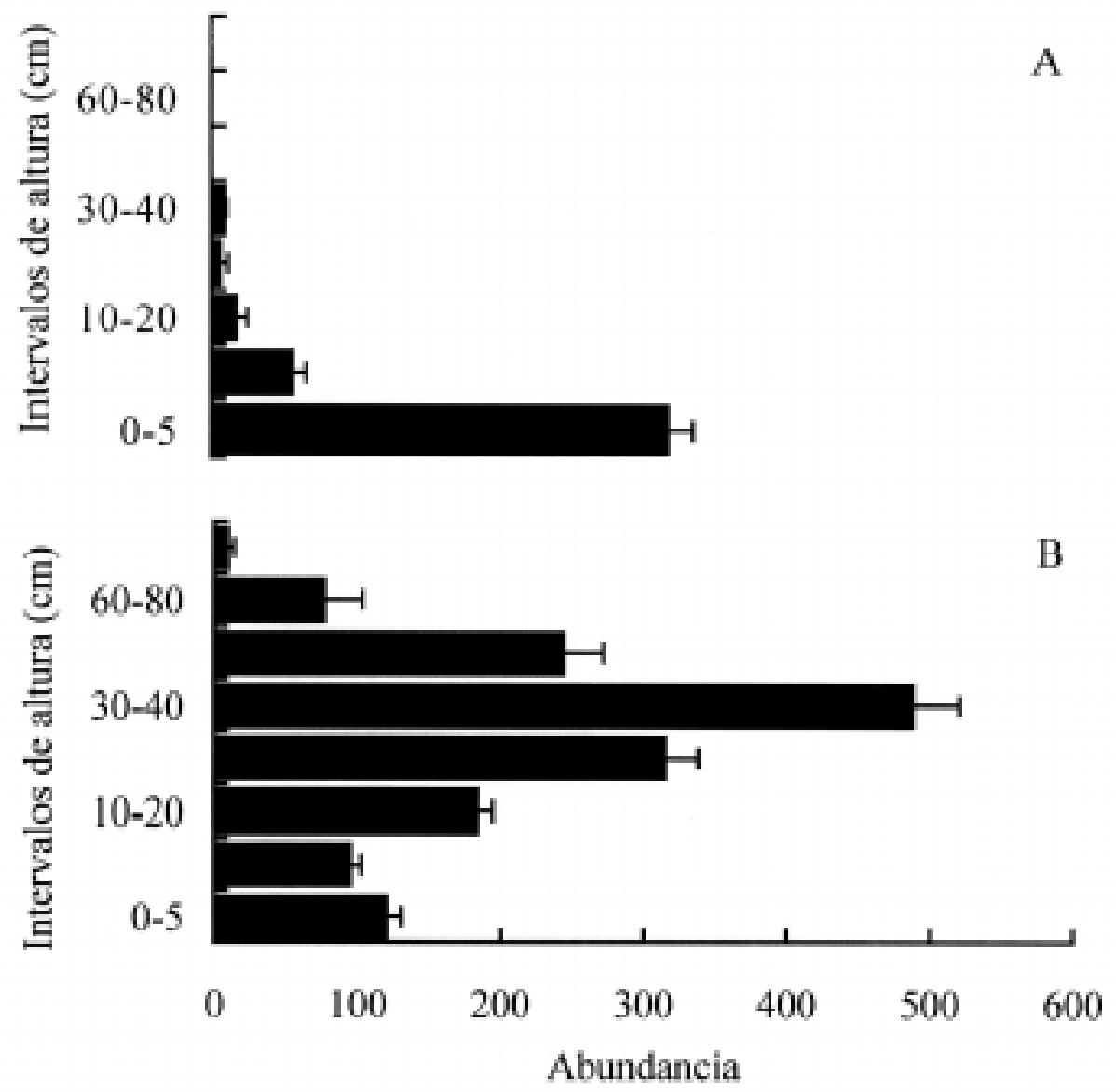

Fig. 3: Abundancia de la vegetación (número de contactos en las agujas +1 error estándar) a diferentes intervalos de altura, en pastizales de montaña de las Sierras de Córdoba, Argentina: (A) tratamiento pastoreado; (B) tratamiento excluido al pastoreo los últimos 10 años.

Vegetation abundance (pin contacts number +1 standard error) in different height intervals, in mountain grasslands of Sierras de Córdoba, Argentina: (A) grazed treatment; (B) exclusion from grazing the last 10 years. 
debajo del suelo (Blomqvist et al. 2000). Los cambios en la estructura vertical observados en el pastizal natural son coincidentes con los registrados por Pucheta et al. (1998) en el mismo área de estudio, quienes observaron que la disminución en la biomasa total de sitios pastoreados está asociada con un incremento relativo de especies pertenecientes a grupos funcionales de graminoides y rastreras, y una disminución del grupo funcional de gramíneas de gran estatura (especies dominantes). Resultados similares se han encontrado en muy diversos sistemas de pastizales en presencia de herbívoros nativos (Milchunas \& Lauenroth 1989, Biondini et al. 1998, Perevolotsky \& Seligman 1998, Knapp et al. 1999) o domésticos (Sala et al. 1986).

Generalmente, los estudios que evalúan los efectos del pastoreo sobre la estructura de pastizales naturales, se han concentrado en la composición florística y en la diversidad de especies de la comunidad (Chaneton \& Facelli 1991, McIntyre \& Lavorel 1994, Anderson \& Briske 1995), pero menores han sido los esfuerzos por entender la relación entre pastoreo, diversidad de especies y diversidad estructural de la vegetación (Sala et al. 1986, Milchunas \& Lauenroth 1989, Pettit et al. 1995), enfoque muy importante a la hora de relacionarlo con otros niveles tróficos diferentes de los grandes herbívoros, como por ejemplo, artrópodos (Gardner et al. 1995, Molina et al. 1999, Rambo \& Faeth 1999).

En los pastizales naturales de Pampa de Achala, el pastoreo por grandes herbívoros a una carga ganadera moderada a intensa permite el mantenimiento de comunidades de céspedes de alta diversidad de especies, sin la invasión de especies exóticas, pero simplifica la estructura vertical y horizontal del sistema, probablemente disminuyendo la disponibilidad de hábitats para otros niveles tróficos.

La presencia conjunta en el espacio de parches excluidos al pastoreo, caracterizados por la dominancia de gramíneas perennes de gran estatura como Deyeuxia hieronymi, Poa stuckertii y Festuca tucumanica, y de parches pastoreados, caracterizados por especies subordinadas (Carex spp., Relbunium richardianum) y transitorias (Muhlenbergia peruviana) (sensu Grime 1998), debería ser tenida en cuenta en planes de manejo de los pastizales de montaña, dado sus diferencias en su respuesta frente a perturbaciones y a cambios climáticos (Grime 1998, Walker et al. 1999).

Aunque actualmente se discute cuál de los componentes de la diversidad tiene mayor importancia determinando el funcionamiento de los ecosistemas (Wilsey \& Potvin 2000), existe un acuerdo más o menos generalizado sobre la im- portancia de la diversidad de especies o de grupos de especies regulando el funcionamiento y el mantenimiento de los servicios de los ecosistemas (MacGillivray et al. 1995, Chapin et al. 2000). Además, el mantenimiento de una alta diversidad de especies nativas podría significar una mayor capacidad de respuesta del ecosistema frente a cambios ambientales o perturbaciones (Chapin et al. 2000, McCann 2000).

En los pastizales estudiados, la presencia en el espacio horizontal de parches pastoreados y excluidos del pastoreo resultaría en el mejor compromiso entre la conservación de la diversidad florística (con mínima diversidad estructural) y la conservación de la diversidad estructural (con mínima diversidad florística).

\section{AGRADECIMIENTOS}

Sebastián Márquez y Guillermo Funes colaboraron activamente en la toma de datos de campo. D. Gurvich enriqueció con sus ideas una versión inicial del manuscrito. El Consejo de Investigaciones Científicas y Tecnológicas de la Provincia de Córdoba (CONICOR) suministró los fondos para la realización del proyecto (Subs. No. 4366/ 97 a E. Pucheta). El Instituto Multidisciplinario de Biología Vegetal (IMBIV) y la Universidad Nacional de Córdoba brindaron todo el apoyo institucional necesario para su realización. El presente trabajo conformó, en parte, la tesina de Licenciatura de M. Nai-Bregaglio.

\section{LITERATURA CITADA}

ANDERSON VJ \& DD BRISKE (1995) Herbivore-induced species replacement in grasslands: is it driven by herbivory tolerance or avoidance? Ecological Applications 5: 1014-1024.

BELSKY AJ (1986) Does herbivory benefit plants? a review of the evidence. American Naturalist 127: 870-892.

BIONDINI ME, BD PATTON \& PE NYREN (1998) Grazing intensity and ecosystem processes in a northern mixed-grass prairie, USA. Ecological Applications 8: 469-479.

BLOMQVIST MM, H OLFF, MB BLAAUW, T BONGERS \& WH VANDERPUTTEN (2000) Interactions between above- and belowground biota: importance for small-scale vegetation mosaics in a grassland ecosystem. Oikos 90: 582-598.

CABIDO M (1985) Las comunidades vegetales de la pampa de Achala. Sierras de Córdoba, Argentina. Documents Phytosociologiques 9: 431-443.

CABRERA AL (1976) Regiones fitogeográficas argentinas. Editorial Acme S.A.C.I., Buenos Aires, Argentina. $85 \mathrm{pp}$. 
CHANETON EJ \& JM FACELLI (1991) Disturbance effects on plant community diversity: spatial scales and dominance hierarchies. Vegetatio 93: 143-155.

CHAPIN FS, ES ZAVALETA, VT EVINER, RL NAYLOR, PM VITOUSEK, HL REYNOLDS, DU HOOPER, S LAVOREL, OE SALA, SE HOBBIE, MC MACK \& S DÍAZ (2000) Consequences of changing biodiversity. Nature 405: 234-242.

COLLINS SL (1987) Interaction of disturbances in tallgrass prairie: a field experiment. Ecology 68: 1243-1250.

DÍAZ S, A ACOSTA \& M CABIDO (1994a) Community structure in montane grasslands of central Argentina in relation to land use. Journal of Vegetation Science 5: 483-488.

DÍAZ S, A ACOSTA \& M CABIDO (1994b) Grazing and the phenology of flowering and fruiting in a montane grassland in Argentina: a niche approach. Oikos 70: 287-295.

FACELLI JM, CM MONTERO \& RJC LEÓN (1988) Effect of different disturbance regimen on seminatural grasslands from the subhumid Pampa. Flora 180: 241-249.

GARDNER SM, M CABIDO, GR VALLADARES \& S DÍAZ (1995) The influence of habitat structure on arthropod diversity in Argentine semi-arid Chaco forest. Journal of Vegetation Science 6: 349-356.

GRIME JP (1998) Benefits of plant diversity to ecosystems: immediate, filter and founder effects. Journal of Ecology 86: 902-910.

HILL MO \& HG GAUCH (1980) Detrended correspondence analysis: an improved ordination technique. Vegetatio 42: 47-58.

HOBBS RJ \& LF HUENNEKE (1992) Disturbance, diversity, and invasion: implication for conservation. Conservation Biology 6: 324-337.

KARKI JB, YV JHALA \& PP KHANNA (2000) Grazing lawns in Terai grasslands, Royal Bardia National Park, Nepal. Biotropica 32: 423-429.

KNAPP AK, JM BLAIR, JM BRIGGS, SL COLLINS, DC HARTNETT, LC JOHNSON \& EG TOWNE (1999) The keystone role of bison in North American tallgrass prairie. BioScience 49: 39-50.

LEÓN RJC, GM RUSCH \& M OESTERHELD (1984) Pastizales pampeanos: impacto agropecuario. Phytocoenologia 12: 201-218.

LUTI R, M SOLÍS, FM GALERA, N MÜLLER, M BERZAL, M NORES, M HERRERA \& JC BARRERA (1979) Vegetación. En: Vázquez J, R Miatello \& M Roqué (eds) Geografía física de la Provincia de Córdoba: 297-368. Editorial Boldt, Buenos Aires, Argentina.

MacGILLIVRAY CW, JP GRIME, SR BAND, RE BOOTH, B CAMPBELL, GAF HENDRY, SH HILLIER, JG HODGSON, R HUNT, A JALILI, JML MACKEY, MA MOWFORTH, AM NEAL, R READER, IH RORISON, RE SPENCER, K THOMPSON \& PC THORPE (1995) Testing predictions of the resistance and resilience of vegetation subjected to extreme events. Functional Ecology 9: 640-649.

MAGURRAN AE (1988) Ecological diversity and its measurement. Chapman and Hall, London, United Kingdom. 179 pp.
McCANN KS (2000) The diversity-stability debate. Nature 405: 228-233.

McINTYRE S \& S LAVOREL (1994) How environmental and disturbance factors influence species composition in temperate Australian grasslands. Journal of Vegetation Science 5: 373-384.

MILCHUNAS DG \& WK LAUENROTH (1989) Threedimensional distribution of plant biomass in relation to grazing and topography in the short grass steppe. Oikos 55: 82-86.

MILCHUNAS DG \& WK LAUENROTH (1993) A quantitative assessment of the effects of grazing on vegetation and soils over a global range of environments. Ecological Monographs 63: 327-366.

MILCHUNAS DG, OE SALA \& WK LAUENROTH (1988) A generalized model of the effects of grazing by large herbivores on grassland community structure. American Naturalist 132: 87-106.

MOLINA SI, GR VALLADARES, S GARDNER \& M CABIDO (1999) The effects of logging and grazing on the insect community associated with a semi-arid chaco forest in central Argentina. Journal of Arid Environments 42: 29-42.

MONTALVO J, MA CASADO, C LEVASSOR \& FD PINEDA (1993) Species diversity patterns in Mediterranean grasslands. Journal of Vegetation Science 4: 213-222.

NORUŠIS MJ (1992) SPSS for Windows. Advanced Statistics Release 5. SPSS Inc., Chicago, Illinois. 580 pp.

NOY-MEIR I, M GUTMAN \& Y KAPLAN (1989) Responses of Mediterranean grassland plants to grazing and protection. Journal of Ecology 77: 290310.

OLFF H \& ME RITCHIE (1998) Effects of herbivores on grassland plant diversity. Trends in Ecology and Evolution 13: 261-265.

PEREVOLOTSKY A \& NG SELIGMAN (1998) Role of grazing in mediterranean rangeland ecosystems. BioScience 48: 1007-1017.

PETTIT NE, RH FROEND \& PG LADD (1995) Grazing in remnant woodland vegetation: changes in species composition and life form groups. Journal of Vegetation Science 6: 121-130.

PUCHETA E \& M CABIDO (1992) Comunidades de pastizales serranos del centro de Argentina y su relación con el uso pasturil. Phytocoenologia 21: 333 346.

PUCHETA E, S DÍAZ \& M CABIDO (1992) The effect of grazing on the structure of a high plateau grassland in central Argentina. Coenoses 7: 145-152.

PUCHETA E, M CABIDO \& S DÍAZ (1997) Modelo de estados y transiciones para los pastizales de altura de las sierras de Córdoba, Argentina. Ecotropicos (Venezuela) 10: 151-160.

PUCHETA E, M CABIDO, S DÍAZ \& G FUNES (1998) Floristic composition, biomass, and aboveground net plant production in grazed and protected sites in a mountain grassland of central Argentina. Acta Oecologica 19: 97-105.

RAMBO JL \& SH FAETH (1999) Effect of vertebrate grazing on plant and insect community structure. Conservation Biology 13: 1047-1054. 
SALA OE, M OESTERHELD, RJC LEON \& A SORIANO (1986) Grazing effects upon plant community structure in subhumid grasslands of Argentina. Vegetatio 67: 27-32.

WALKER B, A KINZIG \& J LANGRIDGE (1999) Plant atribute diversity, resilience, and ecosystem function: the nature and significance of dominant and minor species. Ecosystems 2: 95-113.
WILSEY BJ \& C POTVIN (2000) Biodiversity and ecosystem functioning: importance of species evenness in an old field. Ecology 81: 887-892.

WILSON AD (1990) The effect of grazing on Australian ecosystems. Proceedings of the Ecological Society of Australia 16: 235-244.

Editor Asociado: J. Gutiérrez

Recibido el 25 de enero de 2001; aceptado el 6 de junio de 2002 


\section{ANEXO 1}

Lista ordenada de las réplicas de los tratamientos pastoreado y excluido del pastoreo según la presencia y abundancia de sus especies, en pastizales de montaña de las Sierras de Córdoba, Argentina. Los valores son intervalos de abundancia (número de contactos en las agujas): (1), 1-10; (2), 11-50; (3), 51-100; (4), 101-150; (5), 151-200; (6), 201-250; (7), 251-300; (8), más de 301

Listing of replicates of grazing and exclusion treatments ordered by their species presence and abundance, in mountain grasslands of Sierras de Córdoba, Argentina. Values are abundance intervals (pin contacts number): (1), 110; (2), 11-50; (3), 51-100; (4), 101-150; (5), 151-200; (6), 201-250; (7), 251-300; (8), over 301

Tratamiento

\begin{tabular}{|c|c|c|c|c|c|c|c|}
\hline \multicolumn{3}{|c|}{ Pastoreado } & \multicolumn{4}{|c|}{ Exclusión } & \\
\hline 23 & 4 & 5 & 6 & 7 & & & \\
\hline $25 \quad 24$ & 343 & 34 & 24 & 18 & 20 & & 14 \\
\hline
\end{tabular}

Número de especies

Especies exclusivas de sitios pastoreados

Carex boliviensis van Heurck \& Müll. Argentina

Castilleja pumila (Benth.) Wedd. ex Herrera

Gamochaeta sp.

Luzula excelsa Buchenau

Ophioglossum crotalophoroides Walter var. nanum J.S. Licht. ex Osten

Poa annua L.

Pratia hederacea (Cham.) G. Don

Ranunculus praemorsus Kunth ex D.C.

Stipa neesiana Trin. \& Rupr. var. neesiana

Tagetes filifolia Lag.

Pycreus rivualris (Kunth) Palla

Muhlenbergia peruviana (P. Beauv.) Steud.

Eleocharis albibracteata Nees \& Meyer ex Kunth var. Albibracteata

Plantago myosuros Lam.

Sisyrinchium chilense Hook.

Stipa nidulans $\mathrm{Mez}$

Agrostis glabra (J. Presl) Kunth var. glabra

Sisyrinchium unguiculatum Griseb.

Agrostis sp.

Alchemilla sp.

Bromus brevis Nees ex Steud. subsp. brevis

Cardionema ramosissima (Weinm.) A. Nelson \& J.F. Machr.

Cerastium arvense $\mathrm{L}$.

Mitracarpus megapotamicus (Spreng.) Kuntze

Sellaginella peruviana (Milde) Hieron.

Especies preferenciales de sitios pastoreados

Juncus pallescens Lam. var. achalensis (Barros) Novara

Agrostis montevidensis Spreng. f. montevidensis

Gamochaeta coarctata (Willd.) Kerguélen

Gentianella parviflora (Griseb.) T.N. Ho

Juncus uruguensis Griseb.

Gamochaeta filaginea (DC.) Cabrera

Stenandrium dulce (Cav.) Nees

Eragrostis lugens Nees var. lugens

Eryngium nudicaule Lam.

Galium richardianum (Gillies ex Hook. \& Arn.) Endl. ex

$\begin{array}{lllll}- & 2 & - & - & - \\ 1 & - & 1 & - & - \\ 1 & 1 & - & - & - \\ 1 & - & - & - & - \\ 1 & - & - & - & - \\ 1 & - & - & - & - \\ 1 & 1 & - & - & - \\ - & - & 1 & - & - \\ 1 & - & - & - & - \\ 1 & - & - & - & - \\ 1 & - & 1 & 1 & - \\ 3 & 5 & 3 & 3 & 3 \\ - & - & 1 & 1 & - \\ 1 & 1 & - & 1 & 1 \\ 1 & 1 & - & 1 & 1 \\ - & 2 & - & 2 & 1 \\ 1 & - & - & - & 2 \\ - & - & 1 & 1 & 1 \\ - & - & - & - & 2 \\ - & - & - & - & 1 \\ - & - & - & - & 1 \\ - & - & - & 1 & 1 \\ - & - & - & - & 1 \\ - & - & - & 1 & 1 \\ - & - & - & 1 & 1\end{array}$

Walp. subsp. richardianum 
ANEXO 1 (Continuación)

Tratamiento

Réplica

Número de especies

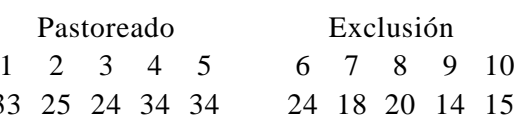

Especies ubicuas

Alchemilla pinnata Ruiz \& Pav.

Poa resinulosa Nees ex Steud.

Carex fuscula d’Urv. var. fuscula f. elatior Kurtz

Taraxacum officinale Weber ex F.H. Wigg.

Briza subaristata Lam.

Oreomyrrhis andicola (Kunth) Hook.

Carex sp.

Sporobolus indicus (L.) R. Br.

Trifolium sp.

Eryngium agavifolium Griseb.

Nothoscordum achalense Ravenna

Oenothera indecora Cambess.

Adesmia bicolor (Poir.) DC.

Noticastrum marginatum (Kunth) Cuatrec.

Sorghastrum pellitum (Hack.) Parodi

Plantago brasiliensis Sims

Margyricarpus pinnatus (Lam.) Kuntze

Chaptalia integerrima (Vell.) Burkart

Adesmia canintosa

Bidens andicola Kunth

Cyperus reflexus Vahl

Dichondra microcalyx (Hallier f.) Fabris

Eryngium ebracteatum Lam.

Grindelia globularifolia Griseb.

Piptochaetium montevidense (Spreng.) Parodi

Especies preferenciales de sitios excluidos al pastoreo

Bromus auleticus Trin. ex Nees

Geranium magellanicum Hook.

Hieracium giganteum Sleumer

Rumex acetosella L.

Deyeuxia hieronymi (Hack.) Türpe

$\begin{array}{llllllllll}4 & 3 & 4 & 2 & 2 & 3 & 3 & 3 & 1 & 1 \\ 2 & 1 & - & 2 & 2 & 2 & 2 & 1 & - & - \\ 1 & 2 & 2 & 1 & 2 & 2 & 3 & 3 & 1 & 1 \\ 1 & 1 & 2 & 1 & 1 & 1 & 1 & 1 & 1 & - \\ 1 & - & 2 & 1 & 1 & 2 & 2 & 2 & 1 & - \\ - & 1 & - & 2 & 2 & 1 & 1 & 1 & 1 & 1 \\ 1 & - & - & 1 & - & 1 & - & - & - & - \\ 2 & - & 1 & 1 & - & 1 & - & 1 & - & - \\ - & 1 & - & - & 1 & - & 1 & - & - & - \\ - & 2 & 2 & - & - & 1 & 1 & - & - & 2 \\ - & 1 & - & - & - & - & - & - & - & 1 \\ - & 1 & - & - & - & - & - & - & - & 1 \\ 1 & - & - & 1 & - & - & - & - & 1 & - \\ - & - & - & 1 & 1 & - & - & - & 1 & 1 \\ - & - & - & 2 & 1 & - & - & - & 2 & - \\ - & - & - & - & 1 & - & - & - & - & 1 \\ - & - & 1 & 1 & - & - & - & 1 & 2 & - \\ 1 & 1 & - & - & - & 1 & 1 & 1 & - & - \\ - & - & - & 1 & - & - & - & 1 & - & - \\ - & - & 1 & - & - & 1 & - & - & - & - \\ 1 & - & - & - & - & - & - & 1 & - & - \\ 1 & - & - & - & - & - & - & 1 & - & - \\ - & - & 1 & - & - & 1 & - & - & - & - \\ - & - & 1 & - & - & 1 & - & - & - & - \\ - & - & 1 & - & - & 1 & - & - & - & -\end{array}$

Especies exclusivas de sitios excluidos al pastoreo

Poa stuckertii (Hack.) Parodi

Festuca tucumanica E.B. Alexeev

Baccharis tucumanensis Hook. \& Arn.

Stipa tenuissima Trin. f. tenuissima

Acicarpha tribuloides Juss.

Hypochaeris argentina Cabrera

Muhlenbergia ligularis (Hack.) Hitchc. 